\title{
Drop-on-Demand E-Jet Printing of Continuous Interconnects with AC-Pulse Modulation on Highly Insulating Substrates
}

\author{
Chuang Wei ${ }^{(a)}$, Hantang Qin ${ }^{(a)}$, Chia-Pin Chiu ${ }^{(b)}$, Yuan-shin Lee ${ }^{(a)}$, Jingyan Dong $^{(a)^{*}}$ \\ (a) Edward P. Fitts Department of Industrial and Systems Engineering \\ North Carolina State University \\ Raleigh, NC, USA \\ (b) Intel Corporation \\ Phoenix, AZ, USA \\ *: To whom all the correspondence should be addressed
}

\begin{abstract}
In this paper, we present a novel AC-pulse modulated electrohydrodynamic (EHD) jet printing technology that enables high resolution fabrication of electrical features and interconnects using the silver nanoink on highly insulating substrates. In traditional EHD jet printing, the remained charge of the printed droplets changes the electrostatic field distribution and interrupts the follow-on printing behavior, especially for highly insulating substrates having a slow charge decay rate. In this paper, a modulated AC-pulsed voltage was used for the EHD jet printing process that can alternate the charge polarity of the consequent droplets to neutralize the residue charge on the substrate. The effect of the residue charge is minimized, which enables high resolution printing of continuous patterns. With three printing parameters (e.g. pulse frequency, voltage, and duration), the EHD jet printing speed and droplet size can be controlled independently. We demonstrated that AC-pulse modulated EHD jet printing can overcome the charge accumulation challenge on highly insulating substrates, and investigated the variables of fabrication process to achieve reliable jet printing of conductive silver tracks.
\end{abstract}

\section{Keywords:}

Electrohydrodynamic (EHD) jet printing, Electrical Interconnects, Drop-on-Demand Printing, AC-pulse modulation,

\section{Introduction}

Printing as a flexible low-cost electronics fabrication method that aims to fabricate electronics directly [1] attracts more attention recently. Ink-jet printing has been widely used for the deposition of a wide range of low viscosity functional materials over large area with high speed [2-3]. The ink-jet printers use acoustic or thermal energy to first form monodispersed droplet in the picoliter volume range, of which the radiuses of the droplet are comparable to the nozzle head. Then the droplets are ejected from the nozzle and be deposited over the desired position on substrates by many methods, such as piezoelectric actuation. Many important electronics features 
have been successfully fabricated by ink-jet printing, for example, electrical interconnections [4], transistors [5-7] and integrated electronic device, organic photovoltaic solar cells [8] and information display [9]. In general, the resolution of the ink-jet printing technique is quite limited. The printing resolution in ink-jet printing is mostly determined by the dimension of the printing nozzle, and is typically limited to about $20 \mu \mathrm{m}$. Reducing the nozzle size with micron-scale orifice to obtain smaller droplets is challenging and costly [2]. Moreover, smaller nozzle size will required a very high and unpractical actuation pressure for many viscous inks, since the printing pressure scales up much faster than the decrease of the nozzle diameter according to Hagen-Poiseuille equation.

Electrohydrodynamic (EHD) jet printing are promising approaches to achieve higher printing resolution [10]. In electrohydrodynamic jet printing, the strong electrostatic field between the nozzle tip and the substrate produces surface charge of the ink and form a Taylor-cone structure, and generate micro-scale droplets [11,12] when the electrostatic stress at the cone tip exceeds the surface tension. The diameter of the droplet is significantly smaller than the nozzle size, which overcome the resolution limitation of the nozzle size. Electrohydrodynamic jet printing has been applied to many applications for printing of conductive tracks[13] and transistors $[14,15]$. However, compared to the wide applicability of ink-jet printing, EHD-jet printing still has some technical difficulties for many applications. In EHD-jet printing, a DC-voltage is applied to the nozzle tip to form the Taylor-cone and the droplets. The printing behavior is mostly determined by the ink properties, printing setup, along with the process conditions [16], such as the applied voltage. Due to the coupled process conditions, the droplet size and the printing frequency/speed cannot be controlled independently.

Besides the limited process controllability, the other critical challenge of EHD jet printing is the residue charge of the droplet from the printing process, which may be stored on the functional surfaces over days or weeks $[17,18]$. The charge on the insulating substrate will change the electrostatic field distribution and the follow-on printing behavior. In EHD jet printing using DC voltage with the same polarity [19-26], the printed droplets on highly insulating substrates retain the same polarity and reject each other[27, 28]. Many highly insulating substrates, such as Teflon and PET, have very slow charge decay rates. The residue charge makes the control of EHD jet printing very challenging for the fabrication of high-resolution continuous features. In many electronic applications, such as flexible electronics and electronic packaging, the commonly used substrates, such as Polyethylene terephthalate (PET) and Ajinomoto Fine film (ABF), are highly insulating and can retain charge for a long time. The residue charge issue needs to be carefully addressed, otherwise will impose heavy constraint on EHD jet printing technology.

In this paper, an AC-pulse modulated electrohydrodynamic (EHD) jet printing process is developed to enable drop-on-demand printing of continuous features on highly insulating substrates (e.g. ABF and PET), which are difficult to be printed with traditional DC-based EHD jet printing process. By using AC-pulsed voltage in printing, the charge of the printed droplets are neutralized by switching of the charge polarity of the consequent 
droplets. The undesired effect of the residue charge is minimized on the EHD printing process, which enables the printing of the high resolution continuous patterns on highly insulating substrates. With three printing parameters (e.g. pulse frequency, voltage, and duration), the EHD jet printing speed and droplet size can be controlled independently. We demonstrated that AC-pulse modulated EHD jet printing can overcome the charge accumulation challenge on highly insulating substrates, and investigated the variables of fabrication process to achieve reliable jet printing of conductive silver tracks.

\section{Printing System and printing Process}

The EHD-jet printing system is composed of three subsystems, as shown in Figure 1: a precision three-axis stage, a pneumatic dispensing system with precision pressure control, and a high voltage supply. The XYZ stage is located on a vibrationisolation table to reduce positioning noise. Three linear stages are configured in XYZ directions with $100 \mathrm{~nm}$ repeatability to move the printing nozzles to the programmed locations. The motion range of the stage is $100 \times 100 \times 50 \mathrm{~mm}$ in XYZ directions. A video camera was used to monitor the printing process.

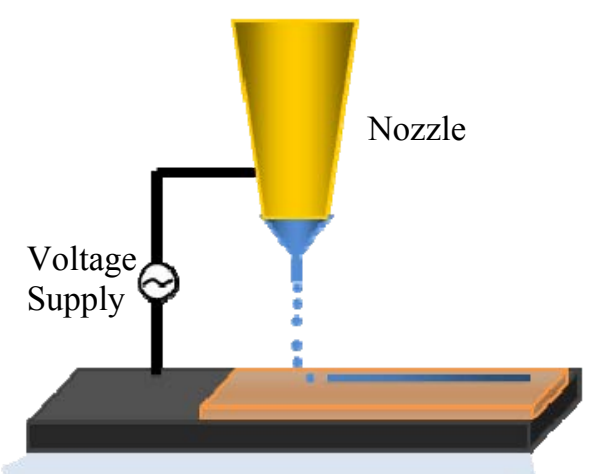

Figure1: Schematic of E-jet printing system. AC pulse voltage is applied between the nozzle and the electrode under the substrate.

The required flow rate of the EHD jet printing is provided by the pressure from a pneumatic syringe, which can provide a maximum pressure of 5 psi with 0.05 psi resolution. The nozzle tip is obtained by pulling a borosilicate capillary and dicing the tip to expose open end under microscope. The substrate electrode is obtained by evaporating $50 \mathrm{~nm}$-thick aluminum on a silicon wafer. The substrates are directly placed on top of the substrate electrode, which is displaced by the XY-stage. A function waveform generator (Agilent 33220A) is used to generate the AC-pulse voltage command signal with programmed voltage, frequency and duration, which is amplified by a high voltage amplifier (Trek 610A) for EHD-jet printing.

AC-pulse voltage signal is used for EHD-jet printing [34-36]. The typical waveform of the command voltage is given in Figure 2. In this AC-pulse modulated EHD-jet printing process, three parameters are used to control the signal and the resulting printing process, which are pulse frequency, pulse voltage amplitude, and pulse duration. The pulse frequency is used to

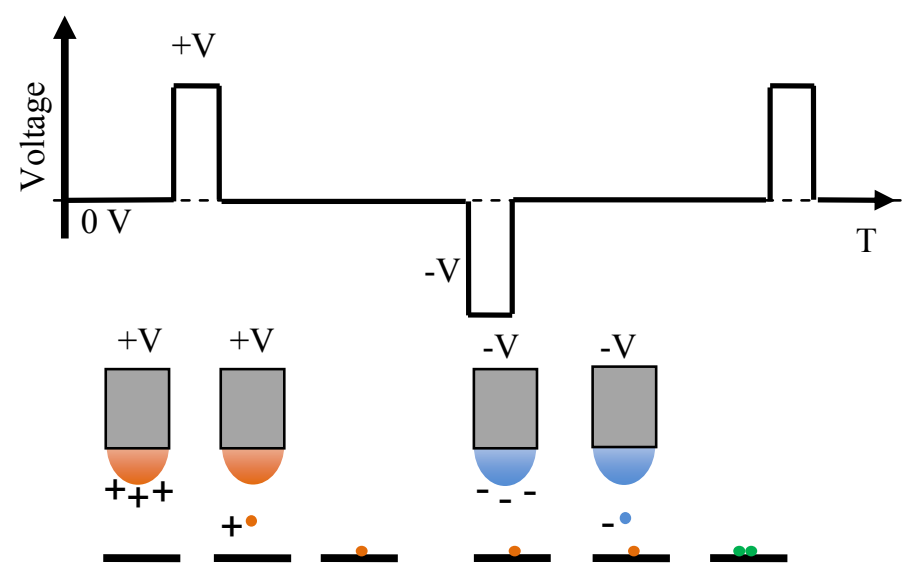

Figure 2: Schematic plot of AC-pulse modulated EHD-jet printing process. Residue charge of printed droplets is neutralized on insulating substrates. 
control the printing speed. When the voltage and the pulse duration are properly selected, each positive or negative pulse will eject a droplet. The resulting printing frequency wills double the pulse frequency of the command signal. The pulse amplitude is selected to provide large enough electrical stress to provide enough electrical field to form the meniscus and eject the droplets. The duration of the voltage pulse determines the time to accumulate charge, which controls the size of the droplets. When each positive and negative pulse eject a droplet, the charge from the consequent droplets neutralizes each other, and effectively minimizes the effect of residue charge.

\section{Characterization of EHD-jet printing process with AC pulsed voltage}

The silver NP ink DGP 40LT-15C (Advanced Nano Products, Co., Ltd, South Korea) with 30-35\% solid content stabilized by proprietary stabilizer is used for EHD printing of continuous interconnects. In the AC-pulse modulated EHD-jet printing process, three parameters that include pulse frequency, pulse voltage, and duty cycle are used to control the printing process. The effect of these process parameters to the printing process was characterized to achieve reliable EHD-jet printing. The process characterization was performed on glass slides. To observe the effect of different process conditions, we changed one process parameter (from pulse frequency, pulse voltage, and duty cycle) at a time while keeping others constant. Then continuous electrical interconnects are directly printed onto the glass substrates, and two highly insulating substrates, Polyethylene terephthalate (PET) and Ajinomoto Fine film (ABF), which are hard to print with DC or DC pulsed voltage.

In EHD-jet printing, the electric stress from the applied voltage deforms the liquid ink at the nozzle tip into the Taylor cone, and eventually produce droplets when the electrical stress is larger than surface tension. Finding the proper voltage range to start the EHD-jet printing process at different the frequency and duty cycle of the pulsed signal is a critical step for process development. In EHD-jet printing, both the electric stress on the meniscus and the duration time when the electric stress is applied are important on the droplet generation. As a result, the required voltage (defining the electrostatic stress) is depend on pulse frequency and duty cycle (together defining the voltage duration time). We experimentally characterized the required voltage amplitude for EHD-jet printing with respect to the pulse frequency and duty cycle. For each fixed duty cycle, using a larger pulse frequency will result in increased threshold voltage to start printing. At a very low pulse frequency, the EHD-printing is very close to the condition where a constant DC voltage is used. At each specific signal frequency, the pulse signal with smaller duty cycle required larger threshold voltage for EHD-jet printing. The reason for this relationship comes from the difference in duration time for different signals. For high frequency pulse or pulse with small duty cycle, with less time for charge accumulation, a larger voltage is needed to produce the meniscus and the droplets. At the high frequency range, we observed a sharp increase in the threshold voltage, which can be explained by the time for charge migration and meniscus formation. The charge will need some time to migrate to the surface of the ink at the nozzle tip to overcome the surface tension and form the meniscus. When 

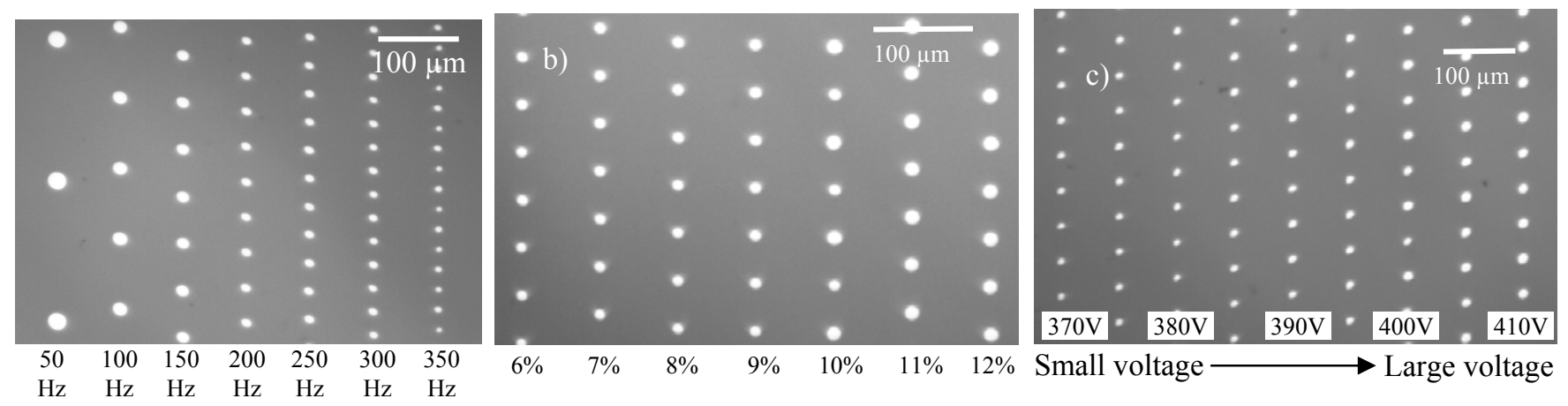

Figure 3: a) printed droplets at different pulse frequencies, and b) at different duty cycles, and c) at different voltage.

the duration time at high frequency is close to the time constant of charge migration, a very large voltage will be needed to provide enough electrical stress to produce the droplets. This time constant of charge migration and meniscus formation generally determines the maximum achievable printing speed.

To find the relationship between pulse frequency and printing speed, we fixed the pulse voltage and the duty cycle, and use different frequency in printing. Given the speed of stage, by measuring the spacing of the printed droplets, the printing frequency can be measured. From experiments, printing frequency is precisely two times of the pulse frequency, which indicates that both positive pulse and negative pulse eject a droplet. The printed droplets have very uniform dimensions (Figure 3(a)), indicating similar charge carried by two consequent droplets, but of opposite polarity. Thus the residue charge can be neutralized in the substrate for continuous feature fabrication. Compared to EHD printing using DC voltage, the process using AC-pulse is robust for insulating substrates, because no net charge is printed onto the substrate surface. For highly insulating substrates used for flexible electronics and electronic sealing and packaging, including ABF, PED, Teflon, and PDMS, charge can stay on the surface for a long time, which make high resolution deposition of continuous interconnection very difficult. As the increase of the printing frequency, a reduced droplet dimension was observed, as shown in Figure 3(a). Since the same duty cycle was used, larger pulse frequency indicates smaller duration time. Thus for the same voltage amplitude used, the produced droplets became smaller due to less time for charge collection at the nozzle tip.

Pulse duty cycle and pulse voltage both affect the droplet size. To characterize the effect of duty cycle on the size of the droplets, a set of printing tests were performed with the pulse frequency and voltage fixed. At very small duty cycle $(<5 \%)$, there is no droplet ejection due to insufficient charge accumulation and electrical stress to produce the droplets. As duty cycle increases from $6 \%$ to $12 \%$, the size of the printed droplets are increased from about $10 \mu \mathrm{m}$ to $14 \mu \mathrm{m}$, as shown in Figure 3(b). The size of the droplets and the duty cycle follows a roughly linear relationship in the range of the duty cycles used in the experiments. For the effect of pulse voltage on the droplet size, Figure 3(c) shows the experimental results on printed droplets at different voltage. It can be clearly found that larger pulse voltage produces larger droplets. The effect of the voltage is similar to the printing results 
based on DC pulsed voltage [19], but is opposite to the results from EHD jet printing with constant DC voltage [29], in which a larger voltage generally results in a higher printing frequency, but smaller droplet size. The difference of the voltage effect may come from the different droplet generation mechanism. The droplet formation mechanism for EHD printing with constant DC voltage is based on tip-streaming [30-32], in which the droplet is generated in unstable region from the Taylor-Cone. For EHD-jet printing using AC pulse voltage, the droplet generation mechanism is very complicated [33]. The retreat of the meniscus when the voltage pulse is removed may produces the droplets, which is similar to the droplet pinch-off in ink-jet printing. Thus larger voltage indicates larger meniscus, and then larger droplets.

\section{Drop-on-Demand Printing of Continuous Interconnects}

To demonstrate the capability and versatility of the process control in AC-pulse modulated EHD-jet printing, a letter " $\mathrm{S}$ " is drop-on-demand printed in the form of separate droplets on glass slides, as shown in Figure 4. The results demonstrate good and independent controllability of the printing speed and droplet size by using AC-pulse modulated EHD-jet printing.

It is very difficult to print continuous features on highly insulating substrate with traditionally EHD-jet printing using constant or pulsed DC voltage, due to the residue charge carried by the printed droplets. For highly insulating substrates, Polyethylene terephthalate (PET) and Ajinomoto

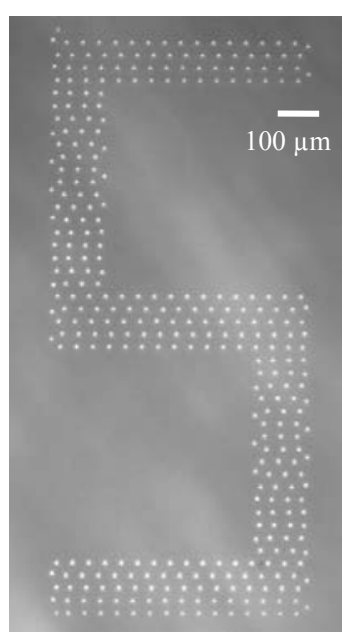

Figure 4: Drop-on-demand printed "S" on glass slides with printing speed and droplet dimension controlled by the parameter of the AC-pulse voltage Fine film (ABF), EHD-jet printing using constant or pulsed DC voltage is uncontrollable which keeps switching between either no printing or printing droplet with huge volume. Previous studies[28] also indicated some other challenges for printing onto highly insulating substrates, such as droplet repulsion, which make the fabrication of continuous features extremely difficult.

Using the AC-pulse modulated EHD-jet printing, we successfully printed droplets with high resolution on ABF and PET substrates, which both are highly insulating substrates with long characteristic time constant for charge decay. However, to achieve continuous interconnects, the separate droplets need to be overlapped properly to form a continuous trace. When the printing speed and droplet size are fixed by certain pulse voltage duration and frequency, the droplet spacing and overlap is purely determined by the plotting speed in which the substrate is moved by the XY-stage. As shown in Figure 5 (a), in order to print connective silver tracks instead of separate dots on substrates, two adjacent droplets has to have a minimum overlap between each other. For high quality interconnects with smooth edge, certain overlap between adjacent droplets has to be used to reduce the edge 

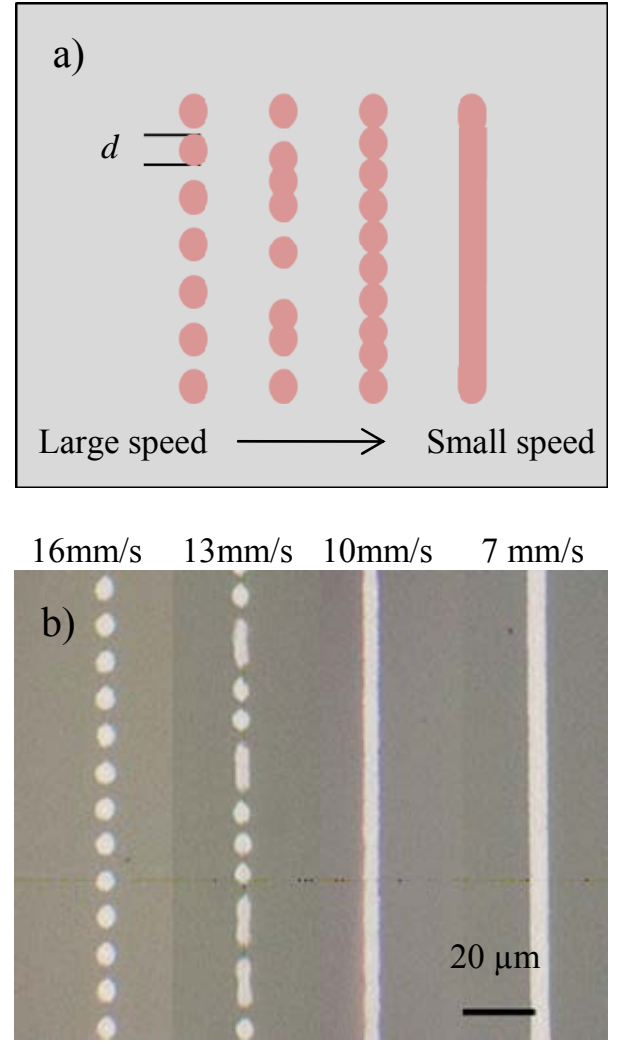

Figure 5: (a) Droplet overlap at different plotting speed. (c) Effect of plotting speeds on the printed interconnects. roughness. As observed from experiments, when the plotting speed is very high $(16 \mathrm{~mm} / \mathrm{sec})$, there is no overlap between adjacent droplets. Separate droplets were printed onto the substrate. As the plotting speed was decreased $(13 \mathrm{~mm} / \mathrm{sec})$, the space between adjacent droplets became smaller. Some of printed droplets are able to be connected with adjacent ones, but the overall tracks are still interrupted. With a proper speed $(10 \mathrm{~mm} / \mathrm{sec})$ and overlap between droplets, we were able to fabricate stable silver lines. When the plotting speed is very slow, there is a large overlap between droplets, continuous silver line was achieved, but with a large line width due to the excess silver ink along the printed line. Figure 5(b) showed silver patterns from discontinuous silver dots to continuous silver tracks with plotting speed decreased from 16 $\mathrm{mm} / \mathrm{s}$ to $7 \mathrm{~mm} / \mathrm{s}$. The diameter of dots reflects the actual resolution of printing system since they are formed by droplets.

Using identified process conditions for AC-pulse modulated EHD-jet printing, we successfully printed continuous interconnects and the contact pads with high resolution on highly insulating substrates, including ABF and PET substrates, as shown in Figure

6. Clearly the contact pad and interconnect line are continuous. The smallest feature dimension (line width) in these patterns is about $3 \mathrm{um}$. These simple continuous features indicate that carried charge in droplets is neutralized on these highly insulating substrates using AC-pulse modulated EHD-jet printing technology; otherwise accumulated residue charge and the resulting repulsion between droplets will make the continuous printing almost impossible.

To obtain good electrical conductivity of the silver lines, a good thickness of the silver lines is required. In
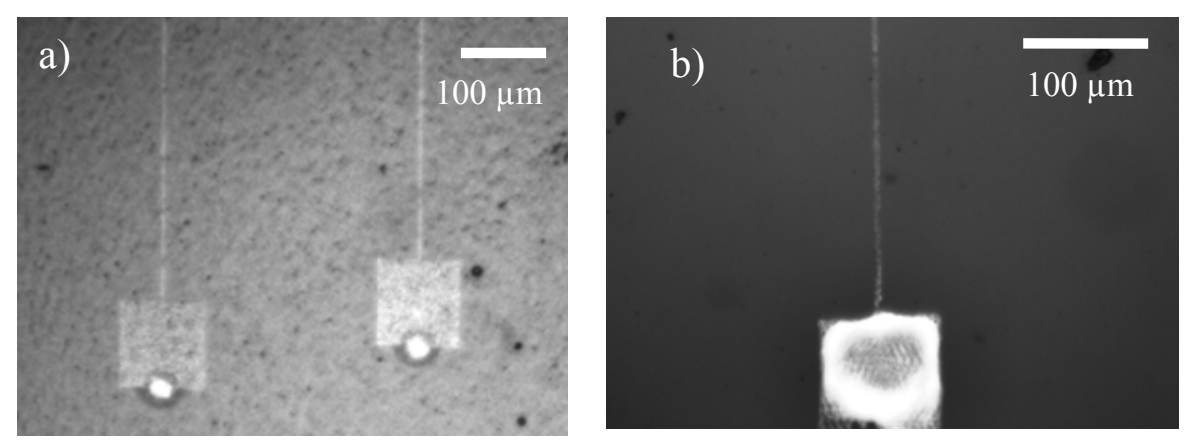
general, to reduce the viscosity of the silver nanoparticle ink, the volume ratio of the silver nanoparticles in the ink solution is relatively small. The thickness of silver line after curing can be very thin for single layer printing, which Figure 6: Continuous features printed on highly insulated surface. a) On ABF indicates relatively poor electrical substrate. b) Features printed on PET film. 

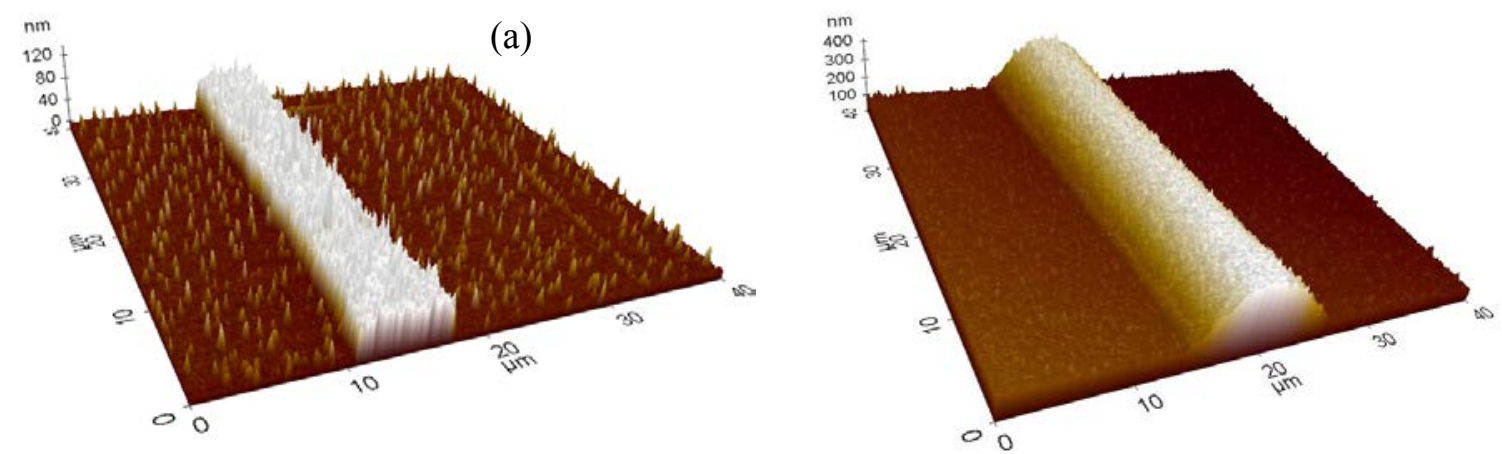

Figure 7: (a) 3D AFM image of silver line with single layer printing, (b) 3D AFM image of silver trace with 20 layers printing.

conductivity. Figure 7(a) shows the AFM images for the printed silver lines. For single layer printing, the maximum height of the cross section of the line is about $60 \mathrm{~nm}$ with an average thickness of $30 \mathrm{~nm}$, which is very close to the diameter of silver nanoparticle. Thus the inter-connectivity of the single layer silver tracks is very poor. By repeating the printing procedure to deposit multi layers for the same pattern, thicker silver trace can be achieved. After printing 20 layers for the silver lines, the fine semi-ellipse cross section was obtained, as shown in Figure 7(b). The maximum height of silver line is measured to be $310 \mathrm{~nm}$ for the 20 layers pattern with an average thickness of $167 \mathrm{~nm}$. By printing the silver ink for multiple layers, line thickness and connectivity were increased with sacrifice of line width. After the curing of silver lines, the 20-layer silver pattern showed good electric conductivity.

\section{Conclusions}

In this paper, a new AC-pulse modulated high-resolution electrohydrodynamic (EHD) jet printing technology was developed and the effect of process parameters on the printing behavior was systematically. By three printing parameters (e.g. pulse frequency, voltage, and duration), the printing speed and droplet size of the EHD jet printing can be controlled independently, in which printing speed is controlled by the pulse frequency, and the droplet size is controlled by the voltage or the pulse duration. By using AC-pulsed voltage, the residue charge of the printed droplets is neutralized by switching of the charge polarity of the consequent droplets. The undesired effect of the residue charge is minimized, which enables high resolution printing of continuous patterns on highly insulating substrates. We demonstrated that AC-pulse modulated EHD jet printing can overcome the residue charge problem for highly insulating substrates, and investigated the parameters of fabrication process to achieve reliable jet printing of conductive silver interconnects.

\section{Acknowledgements}

This work was supported in part by the National Science Foundation under Grant Award CMMI-1129817, CMMI-1333775 and CMMI-1404916 to North Carolina State University. Their support is greatly appreciated. 


\section{References}

[1] Baeg KJ, Caironi M, Noh YY. Toward Printed Integrated Circuits based on Unipolar or Ambipolar Polymer Semiconductors. Adv Mater. 2013.

[2] Basaran OA, Gao H, Bhat PP. Nonstandard Inkjets. Annu Rev Fluid Mech. 2013;45:85-113.

[3] Wong L-Y, Lim G-H, Ye T, Silva F, Zhuo J-M, Png R-Q, Chua S-J, Ho PK. Jettable fluid space and jetting characteristics of a microprint head. J Fluid Mech. 2012;713:109-22.

[4] Van Osch TH, Perelaer J, de Laat AW, Schubert US. Inkjet printing of narrow conductive tracks on untreated polymeric substrates. Adv Mater. 2008;20(2):343-5.

[5] Caironi M, Gili E, Sakanoue T, Cheng X, Sirringhaus H. High yield, single droplet electrode arrays for nanoscale printed electronics. Acs Nano. 2010;4(3):1451-6.

[6] Wang JZ, Zheng ZH, Li HW, Huck WTS, Sirringhaus H. Dewetting of conducting polymer inkjet droplets on patterned surfaces. Nature Materials. 2004;3(3):171-6.

[7] Minemawari H, Yamada T, Matsui H, Tsutsumi Jy, Haas S, Chiba R, Kumai R, Hasegawa T. Inkjet printing of single-crystal films. Nature. 2011;475(7356):364-7.

[8] Angmo D, Sweelssen J, Andriessen R, Galagan Y, Krebs FC. Inkjet printing of back electrodes for inverted polymer solar cells. Advanced Energy Materials. 2013.

[9] Zheng H, Zheng Y, Liu N, Ai N, Wang Q, Wu S, Zhou J, Hu D, Yu S, Han S. All-solution processed polymer light-emitting diode displays. Nature communications. 2013;4.

[10] Jang-Ung P, Hardy M, Seong Jun K, Barton K, Adair K, Mukhopadhyay DK, Chang Young L, Strano MS, Alleyne AG, Georgiadis JG, Ferreira PM, Rogers JA. High-resolution electrohydrodynamic jet printing. Nature Materials. 2007;6(10):782-9.

[11] Ferraro P, Coppola S, Grilli S, Paturzo M, Vespini V. Dispensing nano-pico droplets and liquid patterning by pyroelectrodynamic shooting. Nat Nanotechnol. 2010 Jun;5(6):429-35.

[12] Galliker P, Schneider J, Rüthemann L, Poulikakos D. Open-atmosphere sustenance of highly volatile attoliter-size droplets on surfaces. Proceedings of the National Academy of Sciences. 2013 July 29, 2013.

[13] Schirmer NC, Ströhle S, Tiwari MK, Poulikakos D. On the Principles of Printing Sub-micrometer 3D Structures from Dielectric-Liquid-Based Colloids. Adv Funct Mater. 2011;21(2):388-95.

[14] Sekitani T, Noguchi Y, Zschieschang U, Klauk H, Someya T. Organic transistors manufactured using inkjet technology with subfemtoliter accuracy. P Natl Acad Sci USA. 2008;105(13):4976-80. 
[15] Min S-Y, Kim T-S, Kim BJ, Cho H, Noh Y-Y, Yang H, Cho JH, Lee T-W. Large-scale organic nanowire lithography and electronics. Nature communications. 2013;4:1773.

[16] Stachewicz U, Dijksman JF, Burdinski D, Yurteri CU, Marijnissen JC. Relaxation times in single event electrospraying controlled by nozzle front surface modification. Langmuir. 2009;25(4):2540-9.

[17] Park J-U, Lee S, Unarunotai S, Sun Y, Dunham S, Song T, Ferreira PM, Alleyene AG, Paik U, Rogers JA. Nanoscale, electrified liquid jets for high-resolution printing of charge. Nano Letters. 2010;10(2):584-91.

[18] Baeg KJ, Noh YY, Ghim J, Lim B, Kim DY. Polarity Effects of Polymer Gate Electrets on Non Volatile Organic Field Effect Transistor Memory. Adv Funct Mater. 2008;18(22):3678-85.

[19] Rahman K, Ali K, Muhammad NM, Hyun M-t, Choi K-h. Fine resolution drop-on-demand electrohydrodynamic patterning of conductive silver tracks on glass substrate. Applied Physics A. 2013:1-8.

[20] Mishra S, Barton K, Alleyne A, Ferreira P, Rogers J. High-speed and drop-on-demand printing with a pulsed electrohydrodynamic jet. J Micromech Microeng. 2010;20(9):095026.

[21] Chen C-H, Saville D, Aksay I. Electrohydrodynamic “drop-and-place” particle deployment. Appl Phys Lett. 2006;88(15):154104--3.

[22] Lee MW, An S, Kim NY, Seo JH, Huh J-Y, Kim HY, Yoon SS. Effects of pulsing frequency on characteristics of electrohydrodynamic inkjet using micro-Al and nano-Ag particles. Exp Therm Fluid Sci. 2013;46(0):103-10.

[23] Wang K, Stark JP. Direct fabrication of electrically functional microstructures by fully voltage-controlled electrohydrodynamic jet printing of silver nano-ink. Applied Physics A. 2010;99(4):763-6.

[24] Lee D-Y, Shin Y-S, Park S-E, Yu T-U, Hwang J. Electrohydrodynamic printing of silver nanoparticles by using a focused nanocolloid jet. Appl Phys Lett. 2007;90(8).

[25] Jang Y, Kim J, Byun D. Invisible metal-grid transparent electrode prepared by electrohydrodynamic (EHD) jet printing. Journal of Physics D: Applied Physics. 2013;46(15):155103.

[26] Lee S, Kim J, Choi J, Park H, Ha J, Kim Y, Rogers JA, Paik U. Patterned oxide semiconductor by electrohydrodynamic jet printing for transparent thin film transistors. Appl Phys Lett. 2012;100(10):102108.

[27] Nguyen VD, Byun D. Mechanism of electrohydrodynamic printing based on ac voltage without a nozzle electrode. Appl Phys Lett. 2009;94(17):173509--3.

[28] Kim B, Kim I, Joo SW, Lim G. Electrohydrodynamic repulsion of droplets falling on an insulating substrate in an electric field. Appl Phys Lett. 2009;95(20):204106--3.

[29] Ganan-Calvo A, Davila J, Barrero A. Current and droplet size in the electrospraying of liquids. Scaling laws. 
J Aerosol Sci. 1997;28(2):249-75.

[30] Collins RT, Jones JJ, Harris MT. Electrohydrodynamic tip streaming and emission of charged drops from liquid cones. Nat Phys. 2008;4(2):149-54.

[31] Ganan-Calvo AM. On the general scaling theory for electrospraying. J Fluid Mech. 2004;507(1):203-12a.

[32] Gañan-Calvo AM, Barrero A. The supercritical nature of electrohydrodynamically driven capillary microjets emitted in the EHD spraying of liquids. J Aerosol Sci. 1996;27:S215-S6.

[33] Yeo LY, Lastochkin D, Wang S-C, Chang H-C. A new ac electrospray mechanism by Maxwell-Wagner polarization and capillary resonance. Physical review letters. 2004;92(13):133902.

[34] Chuang Wei, Hantang Qin, N. A. Ramírez-Iglesias, Chia-Pin Chiu, Yuan-shin Lee, Jingyan Dong, "HighResolution AC-Pulse Modulated Electrohydrodynamic Jet Printing on Highly Insulating Substrates”, Journal of Micromechanics and Microengineering, 24(4), 045010 (9pp), 2014.

[35] Hantang Qin, Chuang Wei, Yuan-Shin Lee and Jingyan Dong, "Direct Fabrication of Highly Conductive Micro Silver Tracks Using Electrohydrodynamic Jet Printing with Sub-20 $\mu \mathrm{m}$ Line Width", ASME International Manufacturing Science and Engineering Conference (MSEC 2014), Detroit, June 2014, Paper number (CD): MSEC2014-4163.

[36] Hangtan Qin, Chuang Wei, Jingyan Dong, and Yuan-Shin Lee, "AC-Pulse Modulated Electrohydrodynamic (EHD) Direct Printing of Conductive Micro Silver Tracks for Micro-Manufacturing," Proceedings of The $24^{\text {th }}$. Flexible Automation and Intelligent Manufacturing Conference (FAIM 2014), San Antonio, TX, May 20-23, 2014, Paper number (CD): FAIM2014-231. 